\title{
Aberrant Gcm1 expression mediates Wnt/ $\beta$-catenin pathway activation in folate deficiency involved in neural tube defects
}

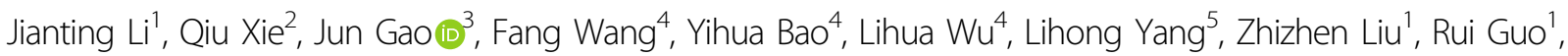 \\ Ajab Khan ${ }^{1}$, Dan Liu', Caihua Li', Jianxin Wu ${ }^{4}$ and Jun Xie (D)
}

\begin{abstract}
Wnt signaling plays a major role in early neural development. An aberrant activation in Wnt/ $\beta$-catenin pathway causes defective anteroposterior patterning, which results in neural tube closure defects (NTDs). Changes in folate metabolism may participate in early embryo fate determination. We have identified that folate deficiency activated Wnt/ $\beta$-catenin pathway by upregulating a chorion-specific transcription factor Gcm1. Specifically, folate deficiency promoted formation of the Gcm1/ $\beta$-catenin/T-cell factor (TCF4) complex formation to regulate the Wnt targeted gene transactivation through Wnt-responsive elements. Moreover, the transcription factor Nanog upregulated Gcm1 transcription in mESCs under folate deficiency. Lastly, in NTDs mouse models and low-folate NTDs human brain samples, Gcml and Wnt/ $\beta$-catenin targeted genes related to neural tube closure are specifically overexpressed. These results indicated that low-folate level promoted Wnt/ $\beta$-catenin signaling via activating Gcm1, and thus leaded into aberrant vertebrate neural development.
\end{abstract}

\section{Introduction}

Neural tube defects (NTDs) are severe birth defects thought to be associated with genetic and environmental factors $^{1}$, resulted from the failure of neural tube closure during first trimester. Prepregnancy supplementation with folate can prevent $30-70 \%$ of $\mathrm{NTDs}^{2}$, but still the mechanism behind its prevention remains unclear.

The Wnt signaling pathway plays a prominent role in gastrulation and anteroposterior axis specification during embryonic development of the nervous system ${ }^{3}$. Wnt1 and Wnt3a are expressed at the dorsal midline of developing neural tube ${ }^{4,5}$. Unnormal levels of $\beta$-catenin in

\footnotetext{
Correspondence: Qiu Xie (xiegiu1999@126.com) or

Jianxin Wu (jianxinwu_2000@163.com) or Jun Xie (junxie@sxmu.edu.cn)

'Department of Biochemistry and Molecular Biology, Shanxi Medical University, 030001 Taiyuan, China

${ }^{2}$ Department of medical research center, Peking Union Medical College Hospital, Chinese Academy of Medical Sciences, 100730 Beijing, China

Full list of author information is available at the end of the article

These authors contributed equally: Jianting Li, Qiu Xie

Edited by A. Verkhratsky
}

embryonic neural stem cells inhibit the proliferation of neural progenitor cells and promote their differentiation ${ }^{6}$. Aberrant canonical Wnt/ $\beta$-catenin pathway signaling leads to defective anteroposterior patterning ${ }^{7}$. The transcriptional activity of genes targeted by Wnt signaling has been proven to be associated with neuronal proliferation and specification ${ }^{6}$. GCM1 (glial cell missing 1), which encodes a chorion-specific transcription factor, has recently been identified as a novel target of $\beta$-catenin/ TCF4 complex during regulation of the fusion of syncytiotrophoblast (ST) cells. The activation of Wnt signaling is essential for upregulation of Gcm1 and ST cell specification. Also, it has been reported that a feedback loop involving $\mathrm{Gcm} 1$ and Frizzled regulates trophoblast differentiation and chorionic branching morphogenesis ${ }^{8}$. Additionally, Wnt10b is involved in the activation of $\beta$-catenin/GCM1 pathway during the process of BeWo cell fusion after forskolin/hCG treatment ${ }^{9}$. These findings suggested that $\mathrm{Gcm} 1$ may be linked with Wnt signaling pathway, which can affect its activity to control cell fate.

\section{(c) The Author(s) 2021}

(c) (i) Open Access This article is licensed under a Creative Commons Attribution 4.0 International License, which permits use, sharing, adaptation, distribution and reproduction cc) in any medium or format, as long as you give appropriate credit to the original author(s) and the source, provide a link to the Creative Commons license, and indicate if changes were made. The images or other third party material in this article are included in the article's Creative Commons license, unless indicated otherwise in a credit line to the material. If material is not included in the article's Creative Commons license and your intended use is not permitted by statutory regulation or exceeds the permitted use, you will need to obtain permission directly from the copyright holder. To view a copy of this license, visit http://creativecommons.org/licenses/by/4.0/. 
Gcm1 gene was first identified as determinant of the glial of Drosophila. In Drosophila, the onset of $\mathrm{gcm}$ expression functions is a binary switch in the developing nervous system and a master regulator of gliogenesis ${ }^{10}$. Further studies identified a DNA-binding domain (the $\mathrm{gcm}$ box) in the amino terminal region of $\mathrm{gcm}$ and a transactivation domain in the carboxy terminal portion ${ }^{11}$. The former is found in target genes that encode transcriptional activators of glial fate and transcriptional repressors of neural fate ${ }^{12}$. Sequence similarities between Drosophila $g \mathrm{~cm} 1$ and mammalian $\mathrm{Gcm} 1$ are high in the $\mathrm{gcm}$ box $^{13}$. Considering its importance in neurogenesis in Drosophila embryos, $\mathrm{Gcm} 1$ was thought to be indispensable for nervous system development in mammals. However, contrary to speculations, rare studies reported mammalian $\mathrm{Gcm}$ genes expression in the nervous system. Instead, $\mathrm{Gcm} 1$ is testified to be essential for human placental development ${ }^{14}$.

In this report, we showed that folate deficiency activates Wnt/ $\beta$-catenin pathway by upregulating $\mathrm{Gcm} 1$ through formation of $\mathrm{Gcm} 1 / \beta$-catenin/TCF4 complex. Moreover, the transcriptional activity of Wnt signaling is regulated through Wnt-responsive elements (WREs). We further showed that Gcm1 is strongly expressed in low-folate NTDs samples, which is accompanied by upregulation of Wnt/ $\beta$-catenin targeted genes related to neural tube closure. Taking together, this study suggested a mechanism by which a signaling pathway can act dynamically to regulate Wnt gene transcriptional programs mediated by $\mathrm{Gcm} 1$ through folate metabolism in neurodevelopment of vertebrates.

\section{Results}

\section{Folate deficiency activates $W n t / \beta$-catenin signaling}

Aberrant Wnt/ $\mathrm{W}$-catenin pathway signaling leads to defective anteroposterior patterning and thus results in $\mathrm{NTDs}^{7}$. To explore the potential effect of folate on Wnt/ $\beta$-catenin pathway in NTDs, a folate-deficient C57BL/6 mESCs model was first established as previously reported $^{15}$. Following sixth generation, no significant differences in the total numbers of cells or cell morphology were observed between the two groups (Fig. 1a). Comparison of cell cycle distribution and apoptosis indicated that arrival at the $G_{2} / M$ checkpoint was not delayed in the sixth generation of folate-deficient group (Fig. 1b), and no difference in apoptosis was observed (Fig. 1c). The critical indicator-folate concentration was with lower intracellular levels in folate-deficient mESCs $\left(1.05 \pm 0.03 \mathrm{ng} / 10^{6}\right.$ cells) than in control mESCs $\left(23.3 \pm 1.78 \mathrm{ng} / 10^{6}\right.$ cells $)$. Additionally, in folate-deficient NE-4C cells for consecutive three generations, the folate concentration in control group was $27.06 \pm 1.52 \mathrm{ng} / 10^{6}$ cells compared with folate-deficient group, which was $1.87 \pm 0.19 \mathrm{ng} / 10^{6}$ cells $(P<0.01$, Fig. $1 \mathrm{~d})$ with no obvious effect on cell survival. Next, we performed an Agilent Mouse mRNA
Array with eight identical arrays per slide $(8 \times 60 \mathrm{~K})$ on folate-deficient C57BL/6 mESCs, focusing on the expression of genes involved in the Wnt/ $\beta$-catenin signaling pathway. Interestingly, real-time RT-PCR showed that $\beta$-catenin upstream genes in the Wnt signaling pathway exhibited no significant transcriptional changes in folate deficiency. However, the downstream target genes of $\beta$-catenin were significantly elevated (0.6-7folds) (Fig. 1e). Meanwhile, the transcriptional activity of TOP/FOP was significantly activated under folate deficiency compared to the positive control group activated by $\mathrm{LiCl}$ (Fig. 1f). Altogether, our data presented the initial evidence that folate deficiency may activate the Wnt/ $\beta$-catenin signaling pathway.

\section{$\mathrm{Gcm} 1$ regulates $W \mathrm{nt} / \beta$-catenin signaling activation by forming the $\mathrm{Gcm} 1 / \beta$-catenin/TCF4 complex}

To identify the potential mechanism of Wnt/ $\beta$-catenin signaling activation with low-folate levels, we first analyzed NTD candidate genes (Table S1) expression through microarray expression profiling. Notably, Gcm1 transcription was ranked the highest among all selected genes under folate deficiency (fold change $>2, P<0.05$, Table S2). The expression of Gcm1 was upregulated 16-folds in folate-free conditions (Fig. 2a). Real-time RT-PCR and western blotting further confirmed that the relative $\mathrm{Gcm} 1$ mRNA expression was significantly higher $(P<0.01$, Fig. $2 \mathrm{~b})$ while the protein level was 2.5 -folds higher $(P<0.05$, Fig. 2c) compared with folate-deficient group. As shown in the database of the gene annotation portal BioGPS, the level of $\mathrm{Gcm} 1$ in normal mESCs is very low, which is consistent with our results (Fig. S1A). Considering that $\mathrm{Gcm} 1$ regulates cell fusion associated with $\mathrm{Wnt} / \beta$-catenin signaling in syncytiotrophoblast cells ${ }^{16,17}$, we therefore investigated to know whether increased $\mathrm{Gcm} 1$ expression regulates Wnt/ $\mathrm{W}$-catenin in folate deficiency. Knockdown of $\mathrm{Gcm} 1$ showed that when $60 \%$ of the $\mathrm{Gcm} 1$ was inhibited $(P=0.03)$, the expression of $\beta$-catenin remained unchanged $(P=0.7)$, while the production of Axin2 and TCF4 were dramatically declined $\sim 64 \%$ and $70 \%$, respectively (for both $P<0.05$, Fig. $2 \mathrm{~d}$, left panel). These results suggested that $\mathrm{Gcm} 1$ has affected the Wnt/ $\beta$-catenin pathway activation, not by affecting the accumulation of $\beta$-catenin in nucleus but by preliminarily influencing TCF4 expression in the nucleus. For further confirmation, TCF4-siRNA parallel experiment was performed. The results corfirmed successful knockdown of TCF4, Gcm 1 , and Axin 2 by $60 \%, 50 \%$, and $55 \%$, respectively, compared with the scramble control group (both $P$ $<0.05$, Fig. $2 \mathrm{~d}$, right panel). Similar results were also verified in folate-deficient NE-4C, with Gcm1 and TCF4 knockdown, which showed reduction in the expression of Axin2 while $\beta$-catenin was remained unchanged (Fig. 2e). The above data revealed that there was a feedback loop 


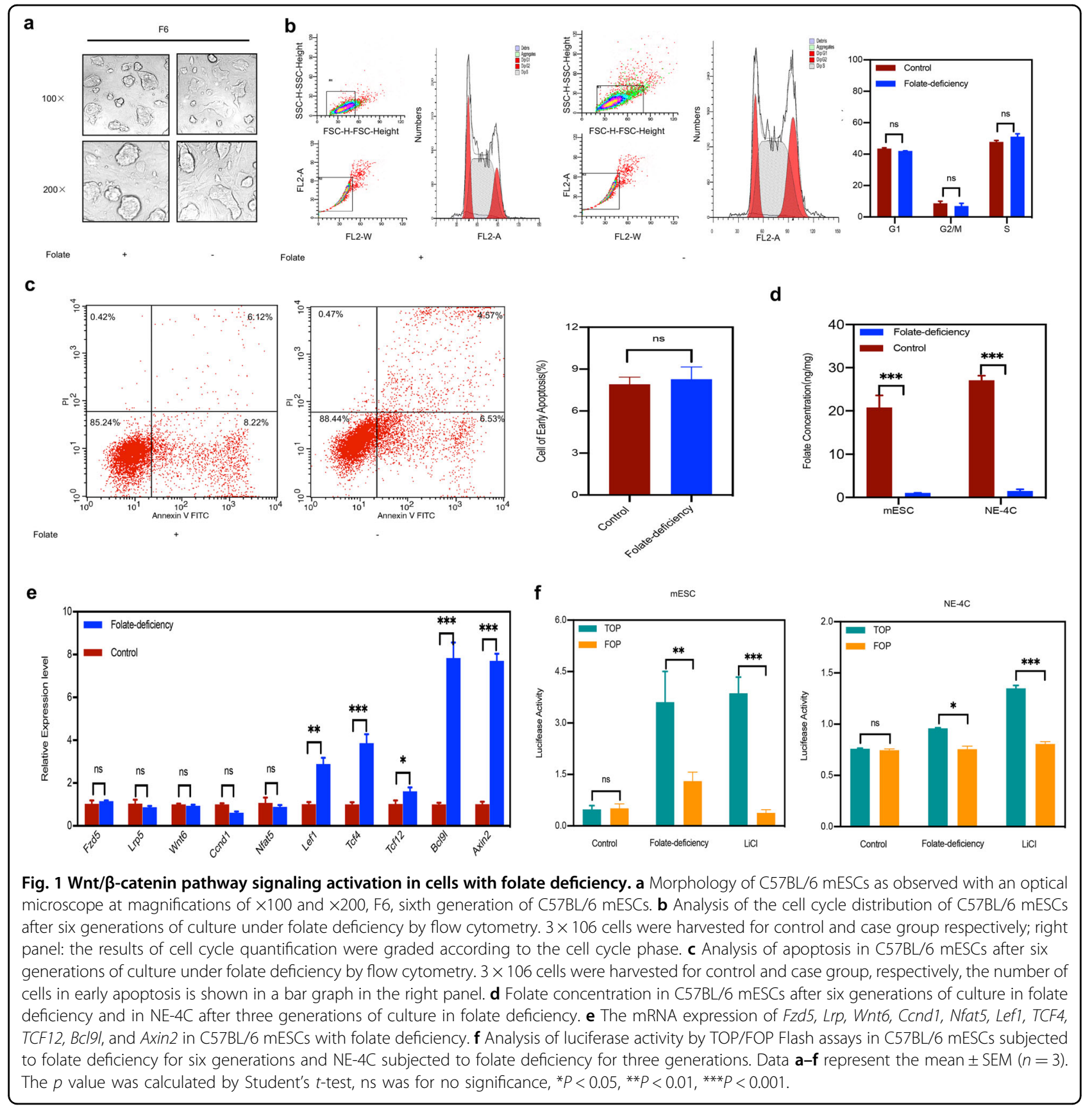

between Gcm1 and TCF4 expression level, which was independent of $\beta$-catenin accumulation in the nuclei with folate deficiency.

To specifically understand the regulation of $\mathrm{Gcm} 1$ in Wnt signaling activation with folate deficiency, coimmunoprecipitation (Co-IP) experiments were performed to explore the possible interactions among $\mathrm{Gcm} 1$, $\beta$-catenin and TCF4. Firstly, we found the level of $\beta$-catenin protein was unchanged in folate deficiency $(P=0.68$, Fig. S1B). Remarkably, Co-IP assay showed a weak and strong $\mathrm{Gcm} 1 / \beta$-catenin binding in the control and folate-deficient groups, respectively, (Fig. 2f, left panel). Since it has been confirmed that TCF4 plays a pivotal role in the activation of $\mathrm{Wnt} / \beta$-catenin pathway $^{18,19}$, we also performed Co-IP assay between Gcm1 and TCF4 in folate deficiency. The results showed an increase in binding between $\mathrm{Gcm} 1$ and TCF4 in folate deficiency (Fig. 2f, middle panel). To further validate whether there is also binding between $\beta$-catenin and TCF4 in folate deficiency, a Co-IP assay with antibody against TCF4 was performed. The results confirmed a detectable increased in the binding between $\beta$-catenin and TCF4 in 


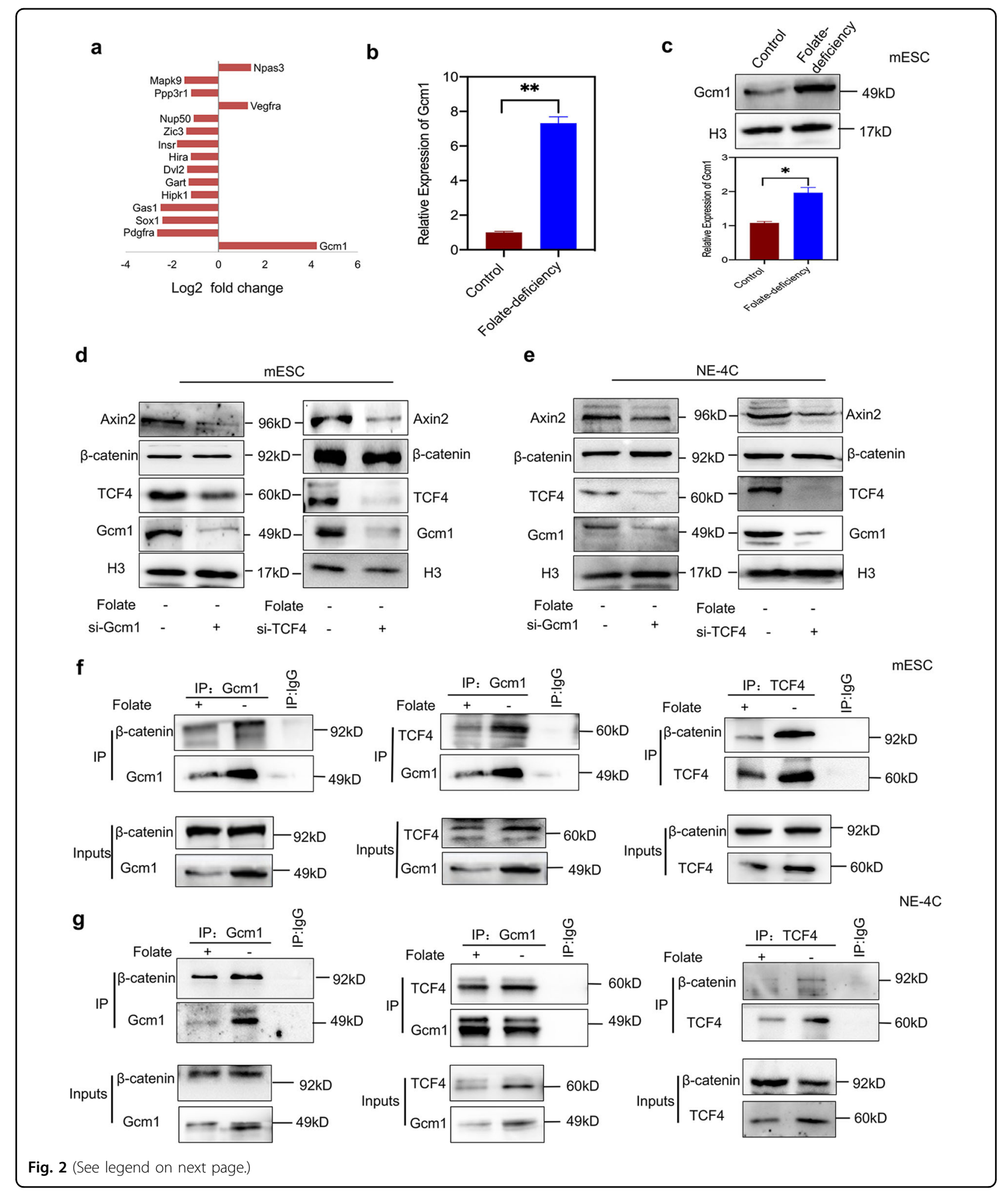

folate deficiency (Fig. 2f, right panel). Similarly, same Co-IP assays in $\mathrm{NE}-4 \mathrm{C}$ revealed an increase in $\mathrm{Gcm} 1$ combining with $\beta$-catenin and TCF4, respectively, in folate-deficient condition (Fig. 2g). Taken together, these results suggested that folate deficiency had significantly regulated the formation of Gcm1, TCF4, and $\beta$-catenin tri-complex. 
(see figure on previous page)

Fig. 2 Folate deficiency upregulates $\mathrm{Gcm} 1$ to promote formation of the $\mathrm{Gcm} \mathbf{1} / \boldsymbol{\beta}$-catenin/TCF4 complex. a Differentially expressed NTD-

related genes in the microarray expression profiles of C57BL/6 mESCs cultured in folate-deficient conditions for six generations. The microarray data were obtained from three replicates for both control and folate-deficient cells. The $X$-axis represents the Log2 fold change. bThe mRNA expression of Gcm 1 in C57BL/6 mESCs after six generations of folate deficiency. ${ }^{* *} P=0.004$. $\mathbf{c}$ Increased expression of Gcm 1 in mESCs with folate deficiency. Top panel: western blots of $\mathrm{G} \mathrm{cm} 1$ in control and folate-deficient cells; bottom panel: bar graph showing the quantification of the western blot signal intensities. $\mathbf{d}$ Efficiency of siRNA-mediated Gcm1 and siRNA-mediated TCF4 depletion and the expression of TCF4, Gcm1 and Axin2 at the protein level in C57BL/6 mESCs. mESCs with folate deficiency were transfected into either scramble siRNA control or Gcm 1 siRNA and TCF siRNA, and post $72 \mathrm{~h}$ of transfection, the expression was determined by western blotting. e Efficiency of siRNA-mediated Gcm 1 and TCF4 depletion and the expression of TCF4 and Axin2 at the protein level in NE-4C. NE-4C with folate deficiency were transfected into either scramble siRNA control or Gcm1 siRNA, TCF4-siRNA, and the expression was determined $72 \mathrm{~h}$ post transfection by western blotting. $\mathbf{f}$ A coimmunoprecipitation (Co-IP) assay was used to identify the interaction between Gcm1, $\beta$-catenin, and TCF4 in C57BL/6 mESC. Immunoprecipitation (IP) was performed using an antiGcm1 antibody and TCF4 antibody, and immunoblotting (IB) was performed using an anti- $\beta$-catenin antibody. g A Co-IP assay was used to identify the interactions between Gcm 1 and TCF4, $\beta$-catenin in NE-4C. An anti-Gcm1 antibody and anti-TCF4 antibody was used for IP, and an anti-TCF4 antibody and anti- $\beta$-catenin was used for IB. Data $\mathbf{b}-\mathbf{f}$ represent the mean $\pm \operatorname{SEM}(n=3)$. The $p$ value was calculated by Student's $t$-test, ns was for no significance, ${ }^{*} P<0.05,{ }^{* *} P<0.01,{ }^{* * *} P<0.001$.

Based on our observation, folate deficiency induced an increased in endogenous Gcm1 expression, which further activated Wnt signaling. Furthermore, we also investigated whether exogenous overexpression of Gcm1 would have a similar effect. Post $72 \mathrm{~h}$ of $\mathrm{Gcm} 1$ plasmid (PGMLV-Gcm1) transfection, both Gcm1 and Axin2 expressions were significantly increased, while $\beta$-catenin expression was remained unchanged $(P=0.53$, Fig. 3a). The Co-IP assay using an antibody against $\mathrm{Gcm} 1$ showed an overexpression of $\mathrm{Gcm} 1$ which promoted self-binding with $\beta$-catenin and TCF4 (Fig. $3 \mathrm{~b}$ and c). Additionally, the interaction between between $\beta$-catenin and TCF4 was significantly increased in the presence of PGMLV-Gcm1 in a dose-dependent manner (Fig. 3d). Lastly, similar strong binding between Gcm1, TCF4, Gcm1, and $\beta$-catenin were also verified in the NE-4C with $\mathrm{Gcm} 1$ overexpression (Fig. 3e). Collectively, all the data demonstrated that overexpression of $\mathrm{Gcm} 1$ promoted Gcm1/ $\beta$-catenin/TCF4 complex formation.

It is well known that $\beta$-catenin binds to TCF4, resulting in the activation of Wnt target gene upon Wnt stimulation ${ }^{19}$.Thus we hypothesized that $\mathrm{Gcm} 1 / \beta$-catenin/TCF4 complex may also play a role in the activation of Wnt signaling when $\mathrm{Gcm} 1$ is overexpressed. Real-time RTPCR demonstrated that the expressions of relative genes (shown in Fig. 1e) were significantly increased (2-folds) (Fig. 3f). The transcription of TOP/FOP exhibited 2.4-fold higher level with Gcm1 overexpression, which was consistent with the positive control group treated with $\mathrm{LiCl}$ (Fig. 3g). Together, these results suggested that an aberrant increased in Gcm1 expression activated Wnt signaling by mediating $\mathrm{Gcm} 1 / \beta$-catenin/TCF4 tri-complex formation.

\section{Gcm1 modulates Wnt target genes coordination with $\beta$-catenin/TCF4 in folate deficiency}

The $\beta$-catenin/TCF4 complex activates Wnt signaling by binding to WREs (5'-CAAAGG-3') in the promoter regions of Wnt target genes to activate their expression ${ }^{19}$. Keeping this in view, we examined whether $\mathrm{Gcm} 1 /$ $\beta$-catenin/TCF4 tri-complex could bind to the promoter WREs of Wnt target genes to control gene expression patterns. ChIP-qPCR experiments with antibodies against $\mathrm{Gcm} 1, \beta$-catenin and TCF4 were performed to assess Wnt targeted genes (Axin2, Bcl9l, and Isl1). A region located $10-\mathrm{kb}$ downstream of the transcription start site (TSS) of the Wnt target gene was examined as a negative control (NEG). As shown in Fig. 4a, ChIP-qPCR demonstrated that folate deficiency enhanced (2-folds) Gcm1 enrichment and (3-folds) $\beta$-catenin enrichment at the WRE sequence of Axin2 in folate-deficient group $(P<0.05)$. Unexpectedly, the enrichment of TCF4 at the WRE of Axin2 was reduced to nearly 5 -fold in folate-deficient group $(P<0.01)$. These results suggested that the binding pattern of the $\mathrm{Gcm} 1 / \beta$-catenin/TCF4 tri-complex in Axin2 changed depending on the folate level. Similar findings were observed for Bcl9l and Isl1 with folate deficiency (Fig. 4b and c). In summary, all these findings revealed that $\mathrm{Gcm} 1 / \beta$-catenin/TCF4 tri-complex specifically bound to WREs of Wnt target genes and orchestrated Wnt target gene activation in folate-deficient conditions.

To explore whether Gcm1 was involved in modulating the transcription of Wnt target genes in folate deficiency, we performed genome-wide ChIP-seq of Gcm 1 in mESCs to determine its binding characteristics. Gene Ontology (GO) analysis showed that the top biological processes overlapped and included developmental processes and nervous system development (Fig. 4d). Notably, PANTHER pathway analysis showed a remarkable number of genes associated with $\mathrm{Gcm} 1$ enrichment were also associated with the Wnt signaling pathway (Fig. 4e). Next, we investigated that how enrichment of $\mathrm{Gcm} 1$ overlapped with Wnt target genes and affect their expression. From ChIP-seq, in the Gcm1 peaks, two Wnt target genes (Ctla4 and Edn1), which are considered as necessary for 


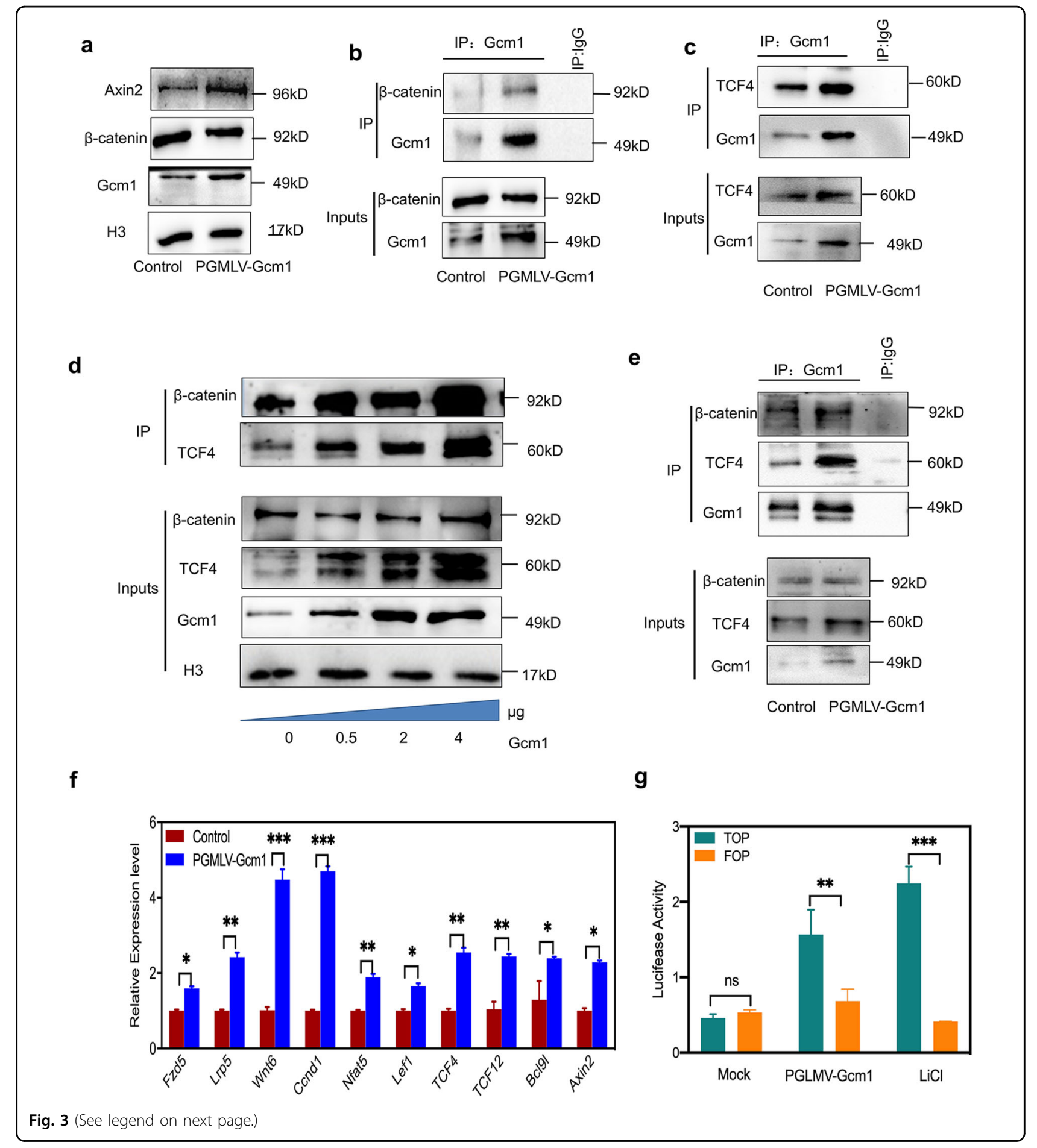

the neural tube closure process, were selected as representatives (Fig. 4f). The WRE sequence of Ctla4 and $E d n 1$ both exhibited decrease in intensity at the $\mathrm{Gcm} 1$ peak in folate deficiency. ChIP-qPCR also verified that Gcm1 interaction at Ctla4 WREs and Edn1 WREs were decreased to approximately $65 \%$ and $61 \%$, respectively
$(P<0.01$, Fig. 4g). Furthermore, the mRNA expression of Ctla 4 and Edn 1 were also ( $72 \%$ and $64 \%$, respectively) lowered in the folate-deficient group $(P<0.01$, Fig. 4 h). Correlation analysis showed a significant positive correlation between $\mathrm{Gcm} 1$ enrichment and Ctla4 and Edn1 mRNA expression (Fig. 4i). These results implied that 
(see figure on previous page)

Fig. $3 \mathrm{Gcm} 1$ overexpression activates the Wnt/ $\beta$-catenin pathway in normal C57BL/6 mESCs and NE-4C. a Increased expression of Axin2 in mESCs with $\mathrm{Gcm} 1$ overexpression. The western blots show that the $\mathrm{Gcm} 1$ overexpression plasmid increased Axin2 expression in normal C57BL/6 mESCs. PGMLV-Gcm1, Gcm1 plasmid. mESCs were transfected with either control siRNA or PGMLV-Gcm1, and the expression levels were determined $72 \mathrm{~h}$ post transfection by western blotting. b A Co-IP assay was used to identify the interaction between $\mathrm{Gcm} 1$ and $\beta$-catenin in cells transfected with pGMLV-Gcm 1 overexpression plasmid in C57BL/6 mESCs. An anti-Gcm1 antibody was used for IP, and IB was performed using an anti- $\beta$-catenin antibody. c A Co-IP assay was used to identify the interaction between Gcm1 and TCF4 in cells transfected with pGMLV-Gcm1 overexpression plasmid in C57BL/6 mESCs. An anti-Gcm 1 antibody was used for IP, and IB was performed using an anti-TCF4 antibody. d A Co-IP assay identified increase interactions between TCF4 and $\beta$-catenin in cells transfected with 0.5, 2, and $4 \mu \mathrm{g}$ of pGMLV-Gcm 1 for $72 \mathrm{~h}$ in C57BL/6 mESCs. e A Co-IP assay was used to identify the interaction between $\mathrm{Gcm} 1$ and TCF4, $\beta$-catenin in NE-4C cells transfected with the pGMLV-Gcm 1 overexpression plasmid. An anti-Gcm 1 antibody was used for IP, and IB was performed using an anti-TCF4 antibody and anti- $\beta$-catenin antibody. $\mathbf{f}$ The mRNA expression of Fzd5, Lrp, Wnt6, Ccnd1, Nfat5, Lef1, Tcf4, Tcf12, Bcl9L, and Axin2 in C57BL/6 mESCs transfected with the overexpression vector pGMLV$\mathrm{Gcm} 1$. The mRNA was extracted from the mESCs after transfection with pGMLV-Gcm 1 for $72 \mathrm{~h}$. $\mathbf{g}$ Analysis of luciferase activity by TOP/FOP Flash assays in C57BL/6 mESCs transfected with pGMLV-Gcm1 overexpression plasmid relative to those transfected with scramble plasmid in normal and $\mathrm{LiCl}$ medium. $\mathrm{LiCl}(10 \mathrm{mmol})$ was added to the control medium before $72 \mathrm{~h}$ of transfection. Data a-g represent the mean \pm SEM $(n=3)$. The $p$ value was calculated by Student's $t$-test, ns was for no significance, ${ }^{*} P<0.05,{ }^{* *} P<0.01,{ }^{* * *} P<0.001$.

Gcm1 enrichment was associated with the transcription of Wnt target genes in folate deficiency. Next, the overall peaks of $\mathrm{Gcm} 1$ were further combined with the microarray data of both control and folate-deficient mESCs. The results conclusively demonstrated that there was a positive correlation between $\mathrm{Gcm} 1$ enrichment and gene expression. Higher Gcm1 enrichment near the TSS region was associated with higher gene expression (Fig. 4j). Overall these data indicated that the $\mathrm{Gcm} 1 / \beta$-catenin/ TCF4 tri-complex bound to Wnt target genes in folate deficiency, in which $\mathrm{Gcm} 1$ had specifically linked to Wnt target promoters.

\section{Nanog upregulates $\mathrm{Gcm} 1$ transcription with folate deficiency}

To understand how folate level affect $\mathrm{Gcm} 1$ expression, we performed the DNA methylation of $G c m 1$ with folate deficiency, considering that folate serves as a source of epigenetic modifications in S-adenosylmethionine (SAM)mediated one-carbon transfer reactions. The results showed that there were no variations in five $\mathrm{CpG}$ sites in the Gcm1 promoter region (Fig. S3A). ChIP-qPCR experiments using antibodies against H3K27me3 and H3K4me3 also showed no differences between the two groups as shown in Fig. S3B.

To further investigate the underlying mechanism of upregulated $\mathrm{Gcm} 1$ transcription in folate deficiency, candidate transcription factor binding motifs near the Gcm 1 promoter were screened. As Nanog binding motifs harboring TBEs are enriched within a 1 kilobase $(\mathrm{kb})$ region of the Gcm1 promoter, the impact of Nanog on Gcm1 gene expression in folate deficiency was investigated (Fig. 5a). Firstly, ChIP-qPCR showed folate deficiency promoted Nanog binding in a region harboring TBE motifs in the promoter of Gcm $1(P<0.05$, Fig. $5 b)$. Next siRNA-Nanog experiment indicated knockdown of Nanog reduced Gcm1 expression at the mRNA level $(P<$ 0.05 , Fig. 5 c). For further confirmation, we tested whether
Nanog upregulates Gcm 1 promoter activity in mESCs, a luciferase assay was performed. As illustrated in Fig. 5d, after co-expression with pGcm1-promoter, Nanog-HA consistently and significantly stimulated the luciferase activity driven by pGcm1-promoter, suggesting that Nanog was required for $\mathrm{Gcm} 1$ promoter activity. In addition, a correlation analysis of folate concentration and Nanog mRNA expression in human NTDs samples also showed a significant negative correlation between them $(P<0.001$, Fig. 5e). However, there was a significant positive correlation between Nanog mRNA expression and Gcm1 mRNA expressions in low-folate NTDs samples, which indicated that Nanog activated the transcription of Gcm1 in low-folate NTDs samples $(P<0.001$, Fig. 5f). Overall, these results demonstrated that Nanog was a critical player in the control of Gcm1 expression with folate deficiency.

\section{Gcm 1 and Wnt target genes upregulation in NTDs}

Now that it was determined that Gcm1 was overexpressed to hyperactivate $\mathrm{Wnt} / \beta$-catenin signaling, we aimed to determine whether the expression of $\mathrm{Gcm} 1$ and Axin2 were affected in NTDs mouse models. The NTDs mouse models used was the same as described previously in our laboratory ${ }^{20,21}$. Mouse nervous system development starts on day 7.5 and get completed on day 10.5 of embryonic development ${ }^{22,23}$ Keeping this in view, neural tissues were examined on day E9.5, because at this time point the nervous development is still in progress. The intact morphology of normal mouse embryos and the morphology of NTDs mouse embryos on day E9.5 were shown in Fig. 6a. Real-time RT-PCR showed that the expression of $\mathrm{Gcm} 1$ was significantly increased in the brains and spines of NTDs mouse embryos (both $P<0.01$, Fig. 6b). Similarly, Axin2 in the brains and spines of NTDs mouse embryos were also increased at mRNA level (both $P<0.05$, Fig. 6c). Notably, the expression of Gcm1 and Axin2 proteins level in the brains and spines of NTDs 


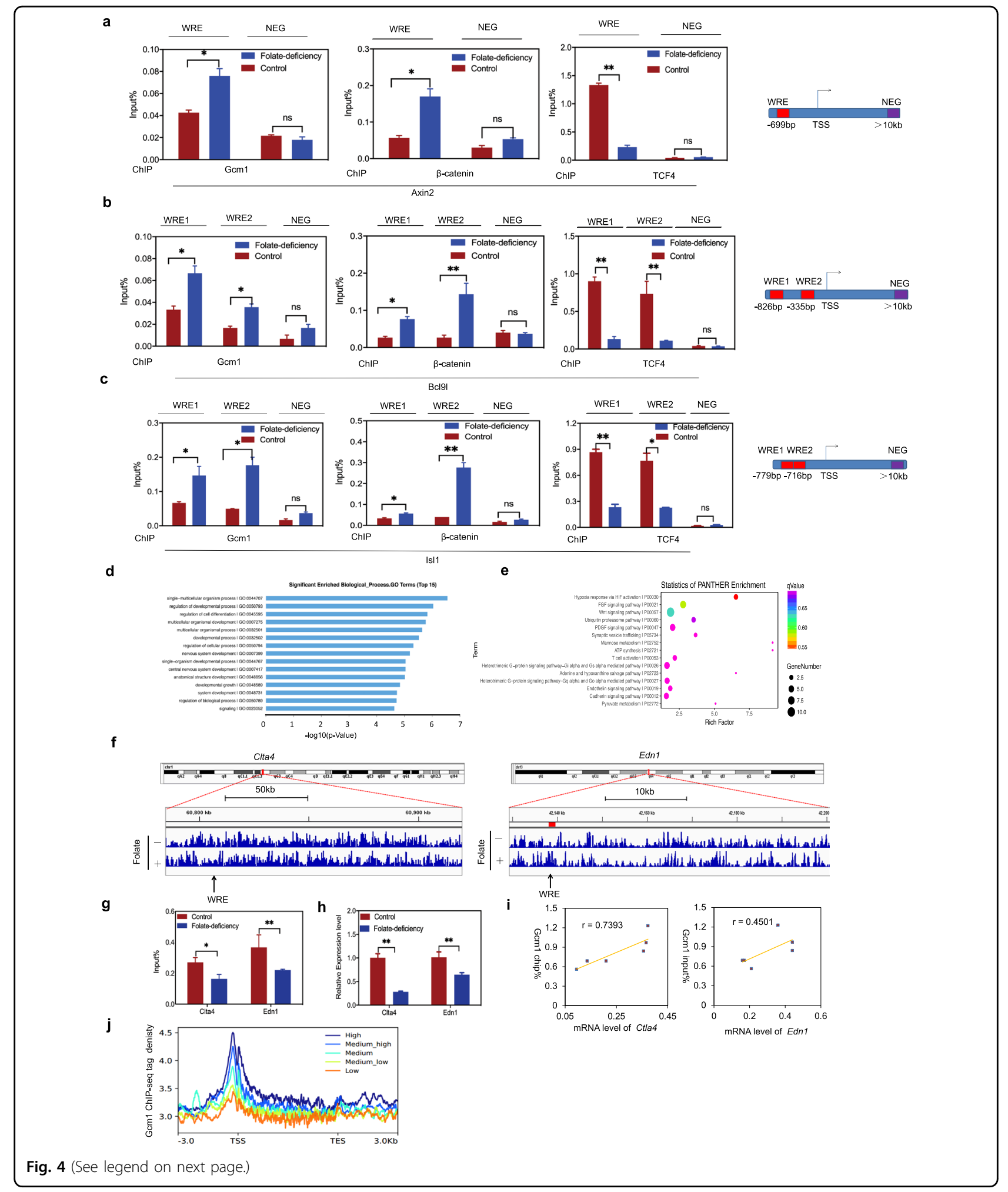

mouse embryos were much higher than that in normal tissue (Fig. $6 \mathrm{~d}$ and e). Finally, the Co-IP assay using an antibody against $\mathrm{Gcm} 1$ showed that an increased binding was found between Gcm1, TCF4, and $\beta$-catenin in NTDs brains and spines (Fig. $6 \mathrm{f}$ and g). All the results confirmed that increased $\mathrm{Gcm} 1$ regulates the Wnt signaling and contributed in neurodevelopment in NTDs mouse models. 
(see figure on previous page)

Fig. $4 \mathrm{Gcm} 1$ competes with $\beta$-catenin for binding to TCF4 and is associated with Wnt target genes expression. a ChIP-qPCR analysis of Gcm 1 , $\beta$-catenin and TCF4 enrichment in Wnt-responsive element (WREs) of Axin2 in control and folate-deficient mESCs. NEG negative control, TSS transcription start site. Right panel: the locations of the WRE and NEG in the promoter. $\mathbf{b}$ ChIP-qPCR analysis of Gcm1, $\beta$-catenin, and TCF4 enrichment in the WREs of BCI9/ in control and folate-deficient mESCs. Right panel: the locations of the WREs and NEG in the promoter. $\mathbf{c}$ ChIP-qPCR analysis of Gcm1, $\beta$-catenin, and TCF4 enrichment in the WREs of $/ s / 1$ in control and folate-deficient mESCs. Right panel: the locations of the WREs and NEG in the promoter. $\mathbf{d}$ Functional clustering of genes associated with Gcm1 ChIP-peaks in mESCs with folate deficiency (the top 10 categories are shown). The $X$-axis represents the $\log 10$ value of the binomial raw $P$ value. e Pathway analysis of $\mathrm{Gcm} 1 \mathrm{ChIP}$-peaks in mESCs with folate deficiency (the top 10 categories are shown). Gcm 1 binding pathway enrichment was determined using PANTHER analysis. The $X$-axis represents the enrichment factor, the $Y$-axis represents the binomial raw $P$ value, and the size of each circle represents the number of genes. $\mathbf{f}$ Representative examples of Ctla4 and Edn1 chromatin binding. A segment of chromosome 1 demonstrates the decreased binding of Clta4 to a TBE in folatedeficient compared to normal mESCs. A segment of chromosome 13 demonstrates the decreased binding of Edn 1 to a TBE in folate-deficient group compared to normal mESCs. $\mathbf{g}$ ChIP-qPCR analysis of Clta4 and Edn1 in mESCs with folate deficiency. ${ }^{*} P=0.015(C l$ ta 4$)$ and ${ }^{*} P=0.022$ (Edn 1). $\mathbf{h}$ The mRNA expression of Clta4 $(\mathrm{J})$ and Edn $1(\mathrm{~K})$ in mESCs with folate deficiency. ${ }^{*} P=0.015$ (Clta4) and ${ }^{*} P=0.04$ (Edn 1). i Correlation analysis between ChIP density and the mRNA expression of Clta4 and Edn1. The correlation coefficients for Clta4 is $0.739(P=0.003)$, and for Edn1 is $0.45(P=0.002)$. $\mathbf{j}$ Correlation of the tag densities ( $Y$-axis) with the transcription levels of genes from $-3 \mathrm{~kb}$ to $+3 \mathrm{~kb}$ ( $X$-axis). All the genes were divided into five groups based on their transcription levels from high (dark blue) to low (orange). The mean-normalized ChIP-seq densities with 1-bp resolution are plotted within a 6-kb region flanking the TSS or the TTS (transcription termination site). Data a-c, $\mathbf{g}-\mathbf{i}$ represent the mean \pm SEM $(n=3)$. The $p$ value was calculated by Student's $t$-test, ns was for no significance, ${ }^{*} P<0.05,{ }^{* *} P<0.01,{ }^{* * *} P<0.001$.

Normal and spina bifida fetal brains (20 each) were also selected and analyzed (Table S3). The folate concentration of normal brains was $0.14 \pm 0.02 \mathrm{ng} / \mathrm{mg}$, while that of spina bifida was $0.06 \pm 0.02 \mathrm{ng} / \mathrm{mg}$ (Fig. 6h). Western blot showed that 5.7 -folds increase in GCM1 level was observed in low-folate NTDs fetuses brain tissue (Fig. 6i). However, GCM1 was minimally expressed in heart, lungs, and muscle tissue (Fig. S4A), which were consistent with the previously reports. The expression of neural tube closure-related genes, Axin $2^{24}$ and Atoh $1^{25,26}$ were analyzed at protein level by western blotting in 40 brain samples. Surprisingly, the expressions of Axin2 and Atoh1 were 1.4-folds and 1.5-folds higher, respectively, in lowfolate NTDs brains (Fig. 6i). Moreover, 12 NTDs samples were used for nanostring experiment, which showed a significant increase in Gcm1, Axin2, and Atoh1 transcription level $(P<0.001$; Fig. $6 j)$. A negative correlation was observed between folate concentration and $\mathrm{Gcm} 1$ mRNA transcription, while the correlations between Gcm1 mRNA transcription with Axin2 mRNA expression, and Atoh 1 mRNA expression were both positive $(P<$ 0.001, Fig. 6k). Lastly, the Co-IP experiment using an antibody against GCM1 revealed an enhanced interaction between GCM1 and $\beta$-catenin in the NTDs brain tissue with low-folate level (Fig. 61). Taken together, these results demonstrated that abnormal expression of GCM1 modulated Wnt/ $\beta$-catenin signaling during the development of mammalian nervous system in folate deficiency.

\section{Discussion}

In mammals, $\mathrm{Gcm} 1$ is abundant in developing placenta and disappears after the completion of placental morphogenesis $^{27}$. In mice, Gcm1 first appears at approximately E7.5 post conception in the chorionic plate, which forms the inner surface of the developing placenta. Upon completion of the placental structure at E10.5, Gcm1 ceases to be expressed. Targeted mutagenesis of Gcm1 gene in mice has been shown to cause embryonic lethality because of the failure of placental development approximately 10 days after conception ${ }^{27}$. It suggests that Gcm1 may function in fundamentally different processes for some cell types and is not limited to being a determinant of glial fate. The chicken ortholog of fly $\mathrm{gcm} 1$ has been demonstrated to be expressed in early neuronal lineages of the developing chick spinal cord ${ }^{12}$. Ectopic expression of $\mathrm{Gcm} 1$ leads to spina bifida in transgenic mice and frequently with a lipoma at the spinal cord, which shared a characteristic with the human spinal dysraphias and suggests human low-axial spina bifida may be related to Gcm1 ectopic expression ${ }^{28}$. However, there was no direct evidence that $\mathrm{Gcm} 1$ directly affects neural specification genes to cause neural tube closure failure.

Our study demonstrate that $\mathrm{Gcm} 1$ contributes to nervous system development in vertebrates. The data suggested that Gcm1-mediated hyperactivation of Wnt/ $\beta$-catenin pathway in folate deficiency resembles the signaling of Wnt3a protein in mESCs, as both mechanisms result in inhibition of the expression of anterior neuroectoderm marker Sox1 (Table S2) ${ }^{29}$. As shown in Figure. $\mathrm{S} 2 \mathrm{~A}$, cluster analysis according to the microarraybased expression profile demonstrated that NTD patterning and embryonic cranial skeleton morphogenesis were significantly affected by folate deficiency. Previous studies have implied that Wnt signaling pathway is involved in anteroposterior axis specification ${ }^{30}$. In the brain and spine of mouse embryos at E8.5, activation of the Wnt/ $\beta$-catenin signaling cascade has been visualized, while Gcm 1 mRNA was undetectable. In fact, the canonical Wnt genes are capable of producing NTDs in mice by hyperactivating WNT, while folic acid supplementation 


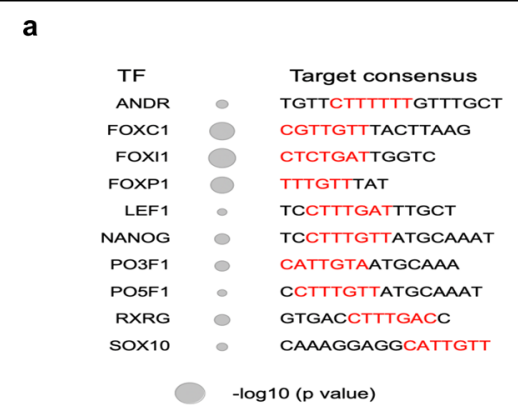

c

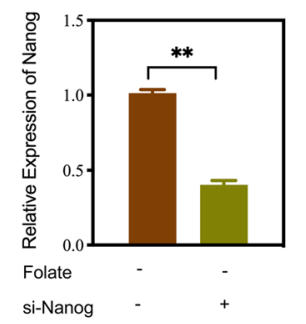

e

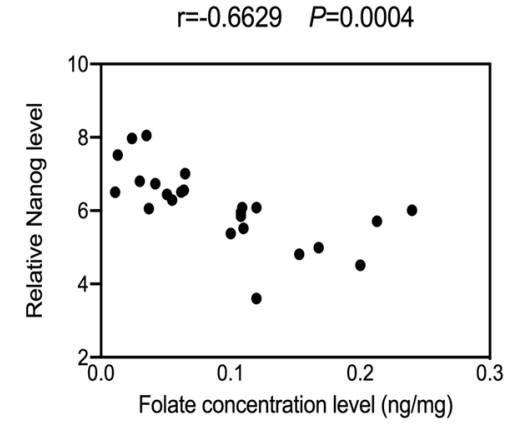

b
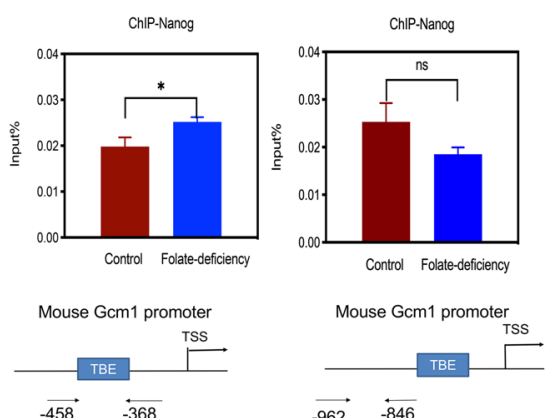

$-4 \overrightarrow{458} \quad-368$

$\overrightarrow{-962} \quad-846$

d

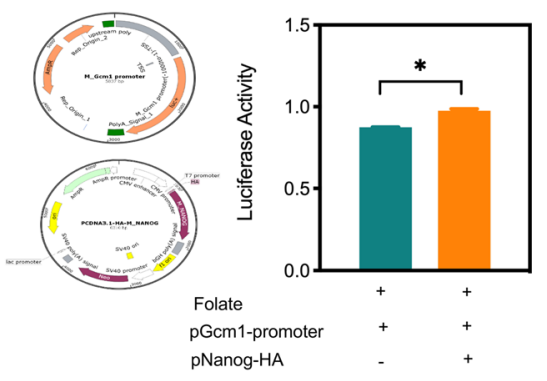

f

$r=0.5642 \quad P=0.0041$

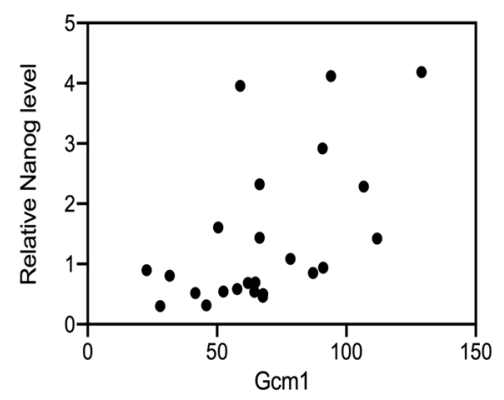

Fig. 5 Nanog upregulates $\mathbf{G} \mathbf{c m} 1$ promoter activity. a Enrichment scores of transcription factor binding motifs in the mouse Gcm 1 promoter. TF transcription factor. The size of the circle represents the $-\log 10$ ( $P$ value). b Nanog recognizes the $\mathrm{Gcm} 1$ promoter. C57BL/6 mESCs were subjected to ChIP analysis using Nanog antibodies. The immunoprecipitated complexes were analyzed by PCR. The TBE sites are located within $1 \mathrm{~kb}$ upstream of the $\mathrm{G} \mathrm{cm} 1$ promoter; the arrows represent the start and end sites of the PCR amplicons (bottom panel). c Efficiency of siRNA-mediated Nanog depletion and the expression of $\mathrm{Gcm} 1$ at mRNA level. C57BL/6 mESCs with folate deficiency were transfected with either control scramble siRNA or Nanog-siRNA, and the expression levels were determined $24 \mathrm{~h}$ post transfection by real-time RT-PCR. Left panel: quantification of Nanog in control scramble siRNA and si-Nanog cells; right panel: quantification of $\mathrm{Gcm} 1$ in control scrambles siRNA and si-Nanog cells. $\mathbf{d}$ Analysis of Gcm1 promoterdriven luciferase reporter activity in folate-deficient mESCs cotransfected with control plasmid or Nanog plasmid. Experimental mESCs were transfected with $1.5 \mu \mathrm{g}$ of $\mathrm{pGcm} 1$-promoter, and control mESCs were transfected with $5.5 \mu \mathrm{g}$ of Nanog-HA. The structures of the Gcm 1 promoter reporter construct and the Nanog-HA construct are shown in the left panel. At $48 \mathrm{~h}$ post transfection, the cells were harvested for luciferase reporter assays. e Correlation analysis of folate concentration and Nanog mRNA expression in low-folate NTDs $(n=12)$. $\mathbf{f}$ Correlation analysis of Nanog mRNA expression and $\mathrm{G} \mathrm{cm} 1 \mathrm{mRNA}$ expression in low-folate NTDs, determined by Nanostring $(n=12)$. Data $\mathbf{b}-\mathbf{d}$ represent the mean \pm SEM $(n=3)$. Data e, $\mathbf{f}$ represent the mean mean $\pm \operatorname{SEM}(n=12)$. The $p$ value was calculated by Student's $t$-test, ns was for no significance, ${ }^{*} P<0.05,{ }^{* *} P<0.01,{ }^{* * *} P<0.001$.

can rescue the mice ${ }^{31}$. These findings indicated a direct relationship between folate level and WNT signaling pathway during neurulation. Our study identified robust expression of Gcm1 and Wnt target genes at E9.5 in both the brains and spines of NTD mouse models. This time and these locations were of critical importance for posterior neuropore closure. Furthermore, increased interaction between $\mathrm{Gcm} 1$ and $\beta$-catenin were found in the brains of NTD fetuses with low-folate levels. It is plausible to believe that overexpression of Gcm1 upregulated Wnt/ $\beta$-catenin signaling activity during neurulation and resulted in the failure of neural tube closure.

To ascertain why Gcm 1 transcription was upregulated in folate deficiency, the possibility that Gcm1 may undergo epigenetic regulation mediated by SAM reactions was investigated. However, no different epigenetic 


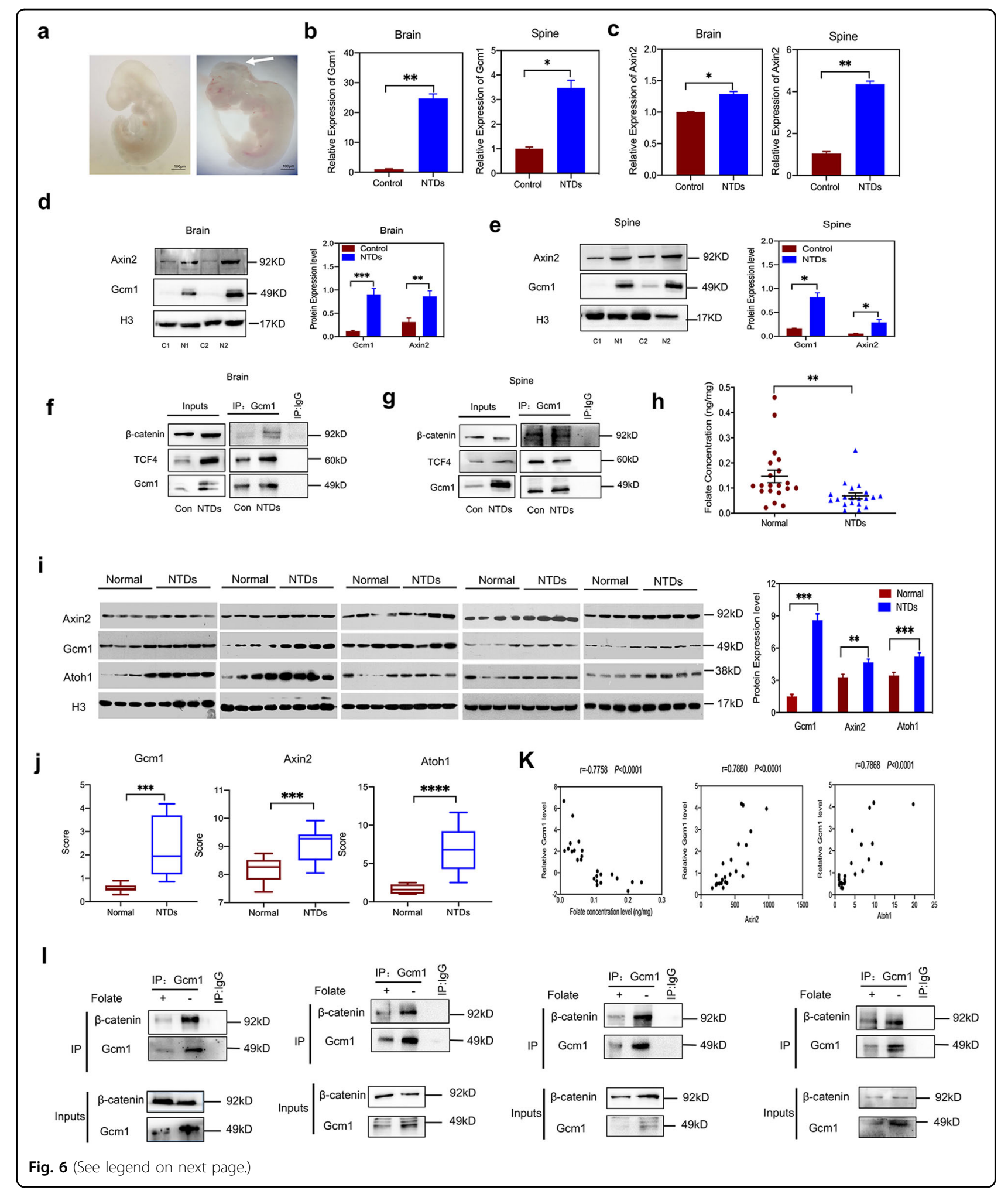

regulation in the promoter region of Gcm 1 was found with folate deficiency. Then, we speculated other effectors. Remarkably, Nanog, which binds with Gcm1 at TBE motifs in the first $1 \mathrm{~kb}$ of the Gcm 1 promoter, can positively regulate $\mathrm{Gcm} 1$ transcription under folate deficiency. In low-folate NTDs samples, a significant negative 
(see figure on previous page)

Fig. $6 \mathrm{Gcm} 1$ and Axin2 are significantly upregulated in NTDs models. a Morphology of CD-1 mouse embryos with low-folate diet and MTX induced from E9.5, left panel is control mouse fetus, right panels are NTDs mouse fetuses. The arrowhead is where the NTDs located; the left panel of NTDs embryos is schizencephaly. Scale bars, $100 \mu \mathrm{m}$. b The mRNA expression of $\mathrm{Gcm} 1$ in the brains and spines of NTD model mice. ${ }^{*} P=0.0003$ (brains) and ${ }^{*} P=0.01$ (spines). $\mathbf{c}$ The mRNA expression of Axin2 in the brains and spines of NTD model mice. ${ }^{*} P=0.002$ (brains) and ${ }^{* *} P=0.02$ (spines). $\mathbf{d}$ Increased expression of Gcm1 and Axin2 in NTD mouse brains. Left panel: western blots of Gcm1 in normal and NTD brains; right panel: bar graph showing the quantification of the western blot signal intensities. e Increased expression of Gcm1 and Axin2 in NTD mouse spines. Left panel: western blots of Gcm1, Axin2 in normal and NTD brains; right panel: bar graph showing the quantification of the western blot signal intensities. f A Co-IP assay was used to identify the interaction between Gcm1 and TCF4, $\beta$-catenin in control and NTDs mouse brains. An anti-Gcm1 antibody was used for IP, and an anti-TCF4 antibody and anti- $\beta$-catenin was used for IB, Con was for Control. $\mathbf{g}$ A Co-IP assay was used to identify the interaction between Gcm1 and TCF4, $\beta$-catenin in control and NTDs mouse spines. An anti-Gcm1 antibody was used for IP, and an anti-TCF4 antibody and anti- $\beta$-catenin was used for IB, Con was for Control. $\mathbf{h}$ Folate concentrations in control human fetal brain tissue and spina bifida human fetal brain tissue. The data were obtained from 40 human fetus brains. i Increased expression of Gcm1, Axin2, and Atoh1 in human NTD brain samples. Left panel: western blots of $\mathrm{Gcm} 1$ in brains of normal humans $(n=20)$ and human NTD $(n=20)$ samples; right panel: bar graph showing the quantification of the western blot signal intensities. $\mathbf{j}$ The mRNA expression of $\mathrm{Gcm} 1$, Axin2, and Atoh1 in the NTDs fetuses, determined by Nanostring $(n=12)$. $\mathbf{k}$ Correlation analysis of folate concentration and $\mathrm{Gcm} 1 \mathrm{mRNA}$ expression (left panel); Gcm 1 mRNA expression and Axin2 mRNA expression, Atoh1 mRNA expression (right panel) $n=12$. I A Co-IP assay identified an interaction between $\mathrm{Gcm} 1$ and $\beta$-catenin in randomly selected low-folate fetal brains. Data $\mathbf{a}-\mathbf{h}, \mathbf{I}$ represent the mean \pm SEM $(n=3)$. Data $\mathbf{i}$ represent the mean \pm SEM $(n=20)$, Data $\mathbf{j}, \mathbf{k}$ represent the mean \pm SEM $(n=12)$, The $p$ value was calculated by Student's $t$-test, ns was for no significance, ${ }^{*} P<0.05,{ }^{* *} P<0.01,{ }^{* * *} P<0.001$.

correlation was observed between folate concentration and Nanog mRNA expression, while the positive correlation between Nanog and Gcm1 mRNA expressions in low-folate NTDs samples indicated that Nanog activated Gcm1 transcription in low-folate NTDs.

It is noteworthy that we characterized Gcm1 overexpression in the brain of NTD-affected fetuses during 15-36 weeks of gestation. The results indicated an unusual $\mathrm{Gcm} 1$ gene transcription, which was maintained over the entire gestational period. Nevertheless. The matching fetal tissues in the first trimeter are less available. This study revealed a previously uncharacterized role of $\mathrm{Gcm} 1$ in regulating Wnt signaling pathway in the development of nervous system during mammal embryogenesis. Based on in vivo and in vitro data, we for the first time demonstrated that $\mathrm{Gcm} 1$ activated $W n t / \beta$-catenin signaling in low-folate levels, which affects neurodevelopment in mammals. These findings will help to understand how spatiotemporal patterning of $\mathrm{Gcm} 1$ regulates and integrates signaling pathways to control cell fate during early mammalian development. In addition, our data will also help to elucidate the mechanism by which folate deficiency causes NTDs during early stages of pregnancy.

\section{Materials and methods}

\section{Cell culture and folate treatment}

C57BL/6 mESCs, KM mouse embryonic fibroblasts (MEFs) and HEK293T cells were kindly provided by the Stem Cell Bank of the Chinese Academy of Sciences (Shanghai, China). NE-4C cells were from ATCC (ATCC number: SCRC-CRL-2925 ${ }^{\mathrm{TM}}$ ) and seeded in a plate that had been precoated with poly-L-lysis (Millipore, USA). Folate power (Sigma-Aldrich, USA) was dissolved in Dulbecco's modified Eagle's medium (DMEM) 10X without folic acid (Sigma-Aldrich, USA) and with 3.7\% filtered sodium hydrogen carbonate (Beijing Chemical Reagent Company, China). The final concentration of $4 \mathrm{mg} / \mathrm{L}$ folate as a normal control while the folatedeficient group was treated simply by DMEM without folate. C57BL/6 mESCs were seeded into culture dishes precoated with MEFs cultured in DMEM supplemented with $0.1 \mathrm{mM}$ nonessential amino acids, $0.1 \mathrm{mM}$ glutamate, and $10 \%$ fetal bovine serum (FBS) (Invitrogen, USA) overnight. The C57BL/ $6 \mathrm{mESCs}$ and NE-4C were cultured in the following complete medium: DMEM with or without $4 \mathrm{mg} / \mathrm{L}$ folate (Sigma-Aldrich, USA), diluted with UltraPure $^{\mathrm{TM}}$ distilled water (Invitrogen, USA), $1 \mathrm{mM}$ glucose, $0.1 \mathrm{mM}$ nonessential amino acids, $0.1 \mathrm{mM}$ glutamate, $\quad 15 \% \quad$ FBS, $0.1 \mathrm{mM} \quad \beta$-mercaptoethanol (Life Technologies, USA), $1000 \mathrm{U} / \mathrm{ml}$ leukemia inhibitory factor (Millipore, USA), and $4 \mathrm{mg} / \mathrm{L}$ folate. The HEK293T cells were seeded in DMEM with 10\% FBS. All cells were maintained at $37^{\circ} \mathrm{C}$ in a humidified atmosphere with $5 \% \mathrm{CO}_{2}$. All cell lines were authenticated by STR profiling and tested as mycoplasma-free.

\section{Microarray expression profiling}

An Agilent Mouse mRNA Array was designed with eight identical arrays per slide $(8 \times 60 \mathrm{~K}$ format $)$ by CapitalBio (Beijing, China), with each array containing probes for approximately 27958 Entrez Gene RNAs and 7419 lncRNAs. The array also contained 1280 Agilent control probes. After six generations of folate deficiency, total RNA was extracted from C57BL/6 mESCs. The RNA was purified, and its concentration was determined based on OD 260/280 readings obtained from a spectrophotometer (NanoDrop ND-1000). RNA integrity was checked by $1 \%$ formaldehyde denaturing gel electrophoresis. cDNA labeled with a fluorescent dye (Cy5 or Cy3-dCTP) was produced by Eberwine's linear RNA 
amplification method and subsequent enzymatic reaction, in accordance with the manufacturer's protocol ${ }^{32}$. Three replicates were designed for each experiment. The array data were summarized, normalized, and subjected to quality control using GeneSpring software V13 (Agilent). To define the differentially expressed genes, we used threshold values of an increased expression of $\geq 2$-folds or a decreased to $\leq 0.5$-fold and a $t$-test $P$ value of 0.05 . The data were log2-transformed and median-centered by gene using the Adjust Data function of Cluster 3.0 software and then further analyzed by hierarchical clustering with average linkage ${ }^{33}$. Finally, we performed tree visualization using Java TreeView (Stanford University School of Medicine, Stanford, CA, USA).

\section{Bisulfite sequencing}

The mESCs samples were treated with bisulfite and the sequences of five $\mathrm{CpG}$ islands and three recognition sites in the mouse $\mathrm{Gcm} 1$ gene were amplified. The CpG islands of the genes were determined using online software (http://emboss.bioinformatics.nl/cgi-bin/emboss/

cpgplot). Primers were designed using the online software Primer3 (http://frodo.wi.mit.edu/cgi-bin)/primer3/primer3_www.cgi). The PCR products were purified with shrimp enzyme (SAP; Promega, USA) and exonuclease I (EXO I; Epicentre) and sequenced using ABI's BigDye 3.1 kit (Cat\# 4323161, USA). The sequencing reactions were purified with alcohol and loaded into an ABI 3730 instrument.

\section{Coimmunoprecipitation (Co-IP) assay}

The Co-IP assay was performed with a kit from Thermo Fisher (Cat. \#26147, USA), following manufacturer's protocol. For immunoprecipitation, $4 \mu \mathrm{g}$ of antibody Gcm1(Santa Cruze, USA) or TCF4 (Santa Cruze, USA) was bounded to Protein A/G plus agarose on a rotator at room temperature for $45 \mathrm{~min}$, with IgG used as a negative control. Then crosslinking of bound antibody with $450 \mu \mathrm{M}$ disuccinimidyl suberate was performed on a rotator at room temperature for $50 \mathrm{~min}$. Simultaneously preclearing of the nuclear protein using control agarose was performed on a rotator at rest at $4{ }^{\circ} \mathrm{C}$ for $50 \mathrm{~min}$. The precleared nuclear protein was incubated with the antibody-crosslinked resin overnight at $4{ }^{\circ} \mathrm{C}$ to accomplish antigen immunoprecipitation. Antigen elution was then completed on the second day and the bounded proteins were detected by western blotting.

\section{RNA extraction, reverse transcription, and real-time RT-PCR analysis}

Total RNA was extracted from $0.5 \times 10^{6}$ cells using TRIzol reagent in accordance with the manufacturer's recommendations (Cat. \#A33254, TRIzol ${ }^{\text {TM }}$ Plus RNA Purification Kit, Invitrogen, USA). Total RNA from various groups were converted into cDNA using TransScript First-Strand cDNA Synthesis SuperMix (TransGen Biotech, China). UltraSYBR Mixture ( $C W$ Biotech, China) was used for real-time PCR, and quantification of the relative mRNA levels in different cell groups, was then performed using a QuantStudio ${ }^{\mathrm{TM}} 7$ Flex Real-Time PCR system (ABI). Primers were synthesized by CWBiotech, which are listed in Table S4. The reactions mixture contained forward and reverse primers $(0.5 \mu \mathrm{l}$ each), UltraSYBR Mixture (12.5 $\mu \mathrm{l}$; containing TransStart Taq, SYBR Green I, dNTPs, and PCR enhancer), cDNA (1 $\mu \mathrm{l})$, and $\mathrm{dd}_{2} \mathrm{O}(10.5 \mu \mathrm{l})$. After the initial denaturation step at $95^{\circ} \mathrm{C}$ for $3 \mathrm{~min}, 45$ amplification cycles were performed as follows: denaturation at $95^{\circ} \mathrm{C}$ for $15 \mathrm{~s}$, annealing at $58^{\circ} \mathrm{C}$ for $20 \mathrm{~s}$, and extension at $72^{\circ} \mathrm{C}$ for $30 \mathrm{~s}$, followed by a final step of $10 \mathrm{~min}$ at $72^{\circ} \mathrm{C}$. The expressions of genes were normalized to that of Gapdh. The relative levels of mRNA transcripts were calculated using the classic $\Delta \Delta \mathrm{Ct}$ method.

\section{Chromatin immunoprecipitation (ChIP) and ChIP-seq}

An Enzymatic Chromatin IP Kit was used for the ChIP assays in accordance with the manufacturer's protocol (Cat. \#9003, SimpleChIP Enzymatic Chromatin IP Kit, Cell Signaling Technology, USA). Formaldehydecrosslinked chromatin was obtained from $\sim 2 \times 10^{7}$ C57BL/6 mESCs after MEFs had been removed. The crosslinked chromatin was immunoprecipitated with antibodies against Gcm1, TCF4, H3K4me3, H3K27me3, and $\beta$-catenin overnight at $4{ }^{\circ} \mathrm{C}$. Nonspecific mouse IgG was used as a negative control.

The immunoprecipitated DNA was analyzed by sequencing. In-depth whole-genome DNA sequencing was performed by CapitalBio Technology (Beijing, China). The raw sequencing data were examined using the illumina analysis pipeline, aligned to the Mus musculus reference genome (University of California, Santa Cruz, mm10) using Bowtie2, and further analyzed with the Model-based Analysis of ChIP-Seq (MACS) program (https://github.com/taoliu/MACS). The enriched binding peaks were identified after normalization with the control input.

\section{Luciferase assay}

A TOPFlash construct $(400 \mathrm{ng}$ ) and a Renilla reporter (400 ng) were separately mixed with a TK control reporter (50 ng) and cotransfected into $10^{6}$ cells by electroporation. For pGLMV-Gcm1 transfection for Gcm1 overexpression, PGLMV-Gcm $1(4 \mu \mathrm{g})$, TOPFlash construct, FOPFlash construct and TK control construct were all mixed together. After $72 \mathrm{~h}$, the Dual-Luciferase ${ }^{\circledR}$ Reporter Assay System (Cat \#E2920, Promega, USA) was used to measure the luciferase activity according to the manufacturer's protocol. The TOP/FOP-flash values were 
normalized to the Renilla reniformis (Promega, USA) reading and the TOP/FOP ratio was measured.

\section{Animals}

CD-1 mice (7-8 weeks old) were provided by Beijing Vital River laboratory Animal Technology Co., Ltd, China (no: 110011200105514958, 16-25 g). Male and female CD-1 mice were fed on low-folate diet for more than 8 weeks. Sexually matured individuals were mated overnight; vaginal plug was detected at 8:00 am in the following morning and was designated as E0.5 day. NTDs mouse models were induced by intraperitoneal injection with $1.5 \mathrm{mg} / \mathrm{kg}$ of MTX (Sigma-Aldrich, USA) on E7.5. On E9.5, pregnant mice were euthanized by cervical dislocation, and the phenotypes of the fetuses were observed under a microscope. All samples with NTDs clinical manifestations were collected for experiments. No specific randomization method was used. All procedures involving animal handling were performed according to the institutional guidelines approved by the Animal Ethics Committee of the Capital Institute of Pediatrics.

\section{Cell cycle and apoptosis analysis by flow cytometry}

For flow cytometry, mESCs were seeded overnight and subjected to folate deficiency for three or six generations. Untreated control cells were simultaneously prepared. C57BL/6 mESCs were harvested, washed, and stained in accordance with the manufacturer's protocol (Cat. \# 340242, BD Cycletest ${ }^{\mathrm{TM}}$ Plus DNA Reagent Kit, Becton Dickinson, USA). The cell cycle phase was analyzed using BD CellQuest Pro software with a FACSCalibur ${ }^{\mathrm{TM}}$ instrument (Becton Dickinson, USA). For apoptosis analyses, mESCs were trypsinized, washed with PBS, resuspended in PBS, and then supplemented with dropwise ice-cold 100\% ethanol to obtain a final ethanol concentration of $75 \%$ for fixation overnight at $-20^{\circ} \mathrm{C}$. The cells were then centrifuged at $1000 \times g$ and $4{ }^{\circ} \mathrm{C}$ for $5 \mathrm{~min}$, washed with PBS, and then resuspended in propidium iodide (PI working solution (PBS containing $100 \mathrm{~g} / \mathrm{ml}$ RNase A, $50 \mathrm{~g} / \mathrm{ml} \mathrm{PI}$, and $0.1 \%$ Triton X-100) for $30 \mathrm{~min}$ in the dark at $4{ }^{\circ} \mathrm{C}$. Finally, equal numbers of cells from control and case groups were filtered through a $35 \mu \mathrm{m}$ strainer cap before being subjected to fluorescence-activated cell sorting (FACS) analysis.

\section{Small interfering RNAs (siRNAs)}

siRNA reagents for $\mathrm{Gcm} 1$ and TCF4 were purchased from Santa Cruz Biotechnology (USA). Nanog-siRNA was purchased from Genomeditech (Shanghai, China). Transfection of cells with Gcm1-siRNA and TCF4-siRNA or Nanog-siRNA was performed by electroporation using the Amaxa ${ }^{\mathrm{TM}}$ Mouse ES Cell NucleofectorTM Kit (Cat. \#VPH-1001, Lonza, Sweden). The control group was transfected with scramble siRNA controls in the same way as mentioned above.

\section{Nanostring}

Total RNA was isolated from 24 human brain tissues following manufacturer's instructions (Cat. \#74104, RNeasy Mini Kits, Qiagen, USA). Gene transcripts analysis and specific probes was conducted using the classic MB subtyping gene CodeSet (Nanostring Technologies, USA). Each reaction contained 500 ng of total RNA in a total of $15 \mathrm{ul}$ aliquot, together with hybridization, reporter, and capture probes. The hybridizations were incubated at $65^{\circ} \mathrm{C}$ for $18 \mathrm{~h}$, then eluted and immobilized in the cartridge on the nCounter Digital Analyzer for data collection. Raw counts were normalized to internal levels for five reference genes including ACTB, TUBB, CLTC, TBP, and POLR1B. Quality control criteria and analysis of the raw Nanostring data were conducted using nSolver Analysis Software v4.0 (Nanostring Technologies, USA). The Student's $t$-test was used to compare normalized expression values between normal and NTDs.

\section{Determination of folate concentrations}

The folate concentration of cells and tissues was determined using a competitive receptor binding immunoassay kit (Cat. \#A14208, Chemiluminescent Immunoenzyme Assay; Beckman Coulter, USA) and an Access 2 Immunoassay System (Beckman Coulter, USA). Briefly, $10^{6}$ cells or $15 \mathrm{mg}$ of brain tissue were collected in $1 \mathrm{ml}$ of Tris buffer solution, sonicated for nine cycles, and centrifuged at $10,000 \mathrm{rpm}$ for $3 \mathrm{~min}$ at $4{ }^{\circ} \mathrm{C}$ before the supernatant was tested.

\section{Subjects}

All clinical samples were obtained from the Lvliang city of Shanxi Province in northern China with informed consent from the patients or their families. The enrolled pregnant women were diagnosed by trained clinicians using ultrasonography. The surgical procedures were performed as previously described ${ }^{34}$. The epidemiological method was described in detail in our previous publication $^{35}$. The Ethics Board of Capital Institute of Pediatrics approved the study protocol. Sample size of human NTDs samples was choosen based on G-power calculation. The information of the clinical samples was shown in Table S3.

\section{Plasmids and antibodies}

All plasmids were purchased from Genomeditech (Shanghai, China). pGLMV-GCM1 was subcloned into a pGMLV-6395 expression vector. pNanog-HA was subcloned into a pcDNA3.1 expression vector. The primary antibodies used were: anti-GCM1 (\#sc-101173, 1:50; Santa Cruz, USA); anti- $\beta$-catenin (\#D1018, 1:1000; CST, USA); anti-Nanog (ab214549,1:1000; Abcam, USA); anti$\beta$-catenin (\#AF0069, 1:1000; Beyotime, China); anti-TCF4 (sc-166699; 1:500, Santa Cruz, USA); anti-Axin2 (\#21515, 
1:2000; CST, USA); anti-histone H3 (\#ab21054, 1:5000; Abcam, USA); and anti-Atoh1 (\#ab168374, 1:1000, Abcam, USA).

\section{Statistical analysis}

All data are presented as the mean \pm SEM. Two-tailed Student's $t$-test was performed to analyze the differences between two groups. Exact sample size(n) is indicated in figure legend of each experiment. All the measurements were repeated at least three times with consistent trends and differences were considered to be statistically significant at ${ }^{*} P<0.05,{ }^{* *} P<0.01,{ }^{* * *} P<0.001$. Data point is excluded if it deviates from mean with more than three standard deviations. No variation is estimated in the data of each group. All statistical analyses were performed using SPSS 22.0 software. Variance is similar between the groups that are being statistically compared. Investigators were not blinded to the group allocation during the experiment and when assessing the outcome in all experiments including animal experiments.

\section{Acknowledgements}

This study was supported by the National Natural Science Foundation of China, (81771584, 81671462, 81701441, 81801453), CAMS Innovation Fund for Medical Sciences (CIFMS, Grant No.2016-I2M-1-008), Public Service Development and Reform Pilot Project of Beijing Medical Research Institute (Grant No. BMR2019-11), National Key Research and Development Program of China (Grant No. 2016YFC1306204), Shanxi Province Key Laboratory of Birth Defects and Cell Regeneration and Research Project Supported by Shanxi Scholarship Council of China, the Fund for Shanxi "1331 Project" Key Subjects Construction, Research Project Supported by Shanxi Scholarship Council of China (2016-051).

\section{Author details}

'Department of Biochemistry and Molecular Biology, Shanxi Medical University, 030001 Taiyuan, China. ${ }^{2}$ Department of medical research center, Peking Union Medical College Hospital, Chinese Academy of Medical Sciences, 100730 Beijing, China. ${ }^{3}$ Department of Postgraduate, Weifang Medical University, 261053 Weifang, China. ${ }^{4}$ Beijing Municipal Key Laboratory of Child Development and Nutriomics, Capital Institute of Pediatrics, 100020 Beijing, China. ${ }^{5}$ Department of Pathology, Shanxi Medical University, 030001 Taiyuan, Shanxi, China. ${ }^{6}$ Department of Bioinformatics, Genesky Biotechnologies Inc, 200120 Shanghai, China

\section{Data availability}

Expression profile microarray data have been deposited in GEO under accession number GSE124271. The ChIP-seq data have been deposited in GEO under accession number GSE124339.

\section{Ethics approval and consent to participate}

All the human samples and animals were approved by the Ethics Board of Capital Institute of Pediatrics. All the human fetal tissues were collected with written consent forms their parents. The committee's reference number for animal is DWLL2018005. For all human fetal information, consent for publication was obtained from their parents.

\section{Conflict of interest}

The authors declare that they have no conflict of interest.

\section{Publisher's note}

Springer Nature remains neutral with regard to jurisdictional claims in published maps and institutional affiliations.
Supplementary Information accompanies this paper at (https://doi.org/ 10.1038/s41419-020-03313-z).

Received: 15 September 2019 Revised: 12 October 2020 Accepted: 14 October 2020

Published online: 04 March 2021

\section{References}

1. Wallingford, J. B., Niswander, L. A., Shaw, G. M. \& Finnell, R. H. The continuing challenge of understanding, preventing, and treating neural tube defects. Science 339, 1222002 (2013).

2. Czeizel, A. E. \& Dudás, I. Prevention of the first occurrence of neural-tube defects by periconceptional vitamin supplementation. N. Engl. J. Med. 327, 1832-1835 (1992)

3. Bielen, H. \& Houart, C. The Wnt cries many: Wnt regulation of neurogenesis through tissue patterning, proliferation, and asymmetric cell division. Dev. Neurobiol. 74, 772-780 (2014).

4. McMahon, A. P. \& Bradley, A. The Wnt-1 (int-1) proto-oncogene is required for development of a large region of the mouse brain. Cell 62, 1073-1085 (1990).

5. Han, M. et al. Folate rescues lithium-, homocysteine- and Wnt3A-induced vertebrate cardiac anomalies. Dis. Model Mech. 2, 467-478 (2009).

6. Selvaraj, P. et al. Neurotrophic factor-a1: a key Wnt- $\beta$-catenin dependent antiproliferation factor and ERK-Sox9 activated inducer of embryonic neural stem cell differentiation to astrocytes in neurodevelopment. Stem Cells 35, 557-571 (2017).

7. Demilly, A., Steinmetz, P., Gazave, E., Marchand, L. \& Vervoort, M. Involvement of the Wnt/B-catenin pathway in neurectoderm architecture in Platynereis dumerilii. Nat. Commun. 4, 1915 (2013).

8. Lu, J. et al. A positive feedback loop involving $\mathrm{Gcm} 1$ and Fzd5 directs chorionic branching morphogenesis in the placenta. PLoS Biol. 11, e1001536 (2013).

9. Malhotra, S. S., Banerjee, P., Chaudhary, P., Pal, R. \& Gupta, S. K. Relevance of Wnt10b and activation of $\beta$-catenin/GCMa/syncytin-1 pathway in BeWo cell fusion. Am. J. Reprod. Immunol. 78, 12676 (2017).

10. Hashemolhosseini, S. \& Wegner, M. Impacts of a new transcription factor family: mammalian GCM proteins in health and disease. J. Cell Biol. 166, 765-768 (2004)

11. Chiu, Y. H. \& Chen, H. GATA3 inhibits GCM1 activity and trophoblast cell invasion. Sci. Rep. 6, 21630 (2016).

12. Soustelle, L. et al. Neurogenic role of $\mathrm{Gcm}$ transcription factors is conserved in chicken spinal cord. Development 134, 625-634 (2007)

13. Yamada, K. et al. Genomic organization, chromosomal localization, and the complete $22 \mathrm{~kb}$ DNA sequence of the human GCMa/GCM1, a placentaspecific transcription factor gene. Biochem. Biophys. Res. Commun. 278, 134-139 (2000).

14. Kojima, J. et al. Efficient production of trophoblast lineage cells from human induced pluripotent stem cells. Lab. Invest. 97, 1188-1200 (2017).

15. Chang, S. et al. Long interspersed nucleotide element-1 hypomethylation in folate-deficient mouse embryonic stem cells. J. Cell. Biochem. 114, 1549-1558 (2013).

16. Zhu, D., Gong, X., Miao, L., Fang, J. \& Zhang, J. Efficient induction of syncytiotrophoblast layer II cells from trophoblast stem cells by canonical Wnt signaling activation. Stem Cell Rep. 9, 2034-2049 (2017).

17. Matsuura, $\mathrm{K}$. et al. Identification of a link between Wnt/ $\beta$-catenin signalling and the cell fusion pathway. Nat. Commun. 2, 1551 (2011)

18. Lu, F. I., Sun, Y. H., Wei, C. Y., Thisse, C. \& Thisse, B. Tissue-specific derepression of TCF/LEF controls the activity of the Wnt/ $\beta$-catenin pathway. Nat. Commun. 5, 5368 (2014).

19. Cadigan, K. M. \& Waterman, M. L. TCF/LEFs and Wnt signaling in the nucleus Cold Spring Harb. Perspect. Biol. 4, a007906 (2012).

20. Wang, $X$. et al. Role of methotrexate exposure in apoptosis and proliferation during early neurulation. J. Appl Toxicol. 34, 862-869 (2014).

21. Massarwa, R., Ray, H. J. \& Niswander, L. Morphogenetic movements in the neural plate and neural tube: mouse. Wiley Interdiscip. Rev. Dev. Biol. 3, 59-68 (2014).

22. $\mathrm{Yu}_{\mathrm{t}} \mathrm{J}$. et al. Transcriptomic profile analysis of mouse neural tube development by RNA-Seq. IUBMB Life 69, 706-719 (2017)

23. Pei, P. et al. Folate deficiency induced H2A ubiquitination to lead to downregulated expression of genes involved in neural tube defects. Epigenetics Chromatin 12, 69 (2019). 
24. Yu, H. M., Liu, B., Costantini, F. \& Hsu, W. Impaired neural development caused by inducible expression of Axin in transgenic mice. Mech. Dev. 124, 146-156 (2007)

25. Machold, R., Klein, C. \& Fishell, G. Genes expressed in Atoh1 neuronal lineages arising from the r1/isthmus rhombic lip. Gene Expr. Patterns 11, 349-359 (2011)

26. Wang, J. et al. Fgf-signaling-dependent Sox9a and Atoh1a regulate otic neural development in zebrafish. J. Neurosci. 35, 234-244 (2015).

27. Baczyk, D. et al. DREAM mediated regulation of GCM1 in the human placental trophoblast. PLOS ONE 8, e51837 (2013).

28. Nait-Oumesmar, B. et al. Ectopic expression of $\mathrm{Gcm} 1$ induces congenital spinal cord abnormalities. Development 129, 3957-3964 (2002).

29. Archer, T. C., Jin, J. \& Casey, E. S. Interaction of Sox1, Sox2, Sox3 and Oct4 during primary neurogenesis. Dev. Biol. 350, 429-440 (2011).

30. Hikasa, H. \& Sokol, S. Y. Wnt signaling in vertebrate axis specification. Cold Spring Harb. Perspect. Biol. 5, a007955 (2013).
31. Carter, M. et al. Crooked tail (Cd) model of human folate-responsive neura tube defects is mutated in Wnt coreceptor lipoprotein receptor-related protein 6. Proc. Natl Acad. Sci. USA 102, 12843-12848 (2005).

32. Luo, L. et al. Gene expression profiles of laser-captured adjacent neuronal subtypes. Nat. Med. 5, 117-122 (1999).

33. Eisen, M. B., Spellman, P. T., Brown, P. O. \& Botstein, D. Cluster analysis and display of genome-wide expression patterns. Proc. Natl Acad. Sci. USA 95, 14863-14868 (1998).

34. Chen, $X$. et al. Detection of copy number variants reveals association of cilia genes with neural tube defects. PLOS ONE 8, e54492 (2013).

35. Chen, X. et al. Global DNA hypomethylation is associated with NTD-affected pregnancy: a case-control study. Birth Defects Res. A Clin. Mol. Teratol. 88 575-581 (2010) 